\title{
QUALIDADE DE VIDA EM PROFESSORES UNIVERSITÁRIOS: UM ESTUDO EM UMA UNIVERSIDADE DO SUL DO TOCANTINS
}

\author{
QUALITY OF LIFE IN UNIVERSITY PROFESSORS: A STUDY AT A UNIVERSITY \\ IN SOUTHERN TOCANTINS
}

Kaique Santana Pinto ${ }^{1}$, Aline Ribeiro Dias ${ }^{2}$, Ítalo Brito Salera ${ }^{3}$, Jeann Bruno Ferreira da Silva ${ }^{4}$, Vinicius Lopes Marinho ${ }^{5}$

\section{RESUMO}

Introdução: De acordo com a OMS (Organização Mundial da Saúde) a Qualidade de Vida (QV) pode ser definida como 'a percepção do indivíduo acerca da sua posição na vida, no contexto da cultura e sistema de valores nos quais ele vive e em relação aos seus objetivos, expectativas, padrões e preocupações". Objetivo: Investigar os a QV de professores universitários de uma universidade do Sul Tocantins. Metodologia: Tratou-se de pesquisa transversal de caráter quantitativa realizada com 85 professores de uma Universidade do Sul do Tocantins, Brasil. Os dados foram analisados por meio da estatística descritiva e de acordo com o manual de correção do WHOQOL-bref, com dados apresentados em gráficos e tabelas. Resultados: Sobre sua QV, $75 \%$ declararam como boa; $14 \%$ nem ruim e nem boa; $9 \%$ classificaram como ruim e $2 \%$ apontaram como muito boa. Considerações finais: Em síntese, a maioria não declarou insatisfação com sua QV. Exceto o escore ambiente, que apontou-se como baixo, acredita-se que os demais são indicadores que refletem o bom relacionamento com alunos, demais colegas e outros aspectos característicos do próprio processo de trabalho.

Palavras-chave: Qualidade de Vida; Docentes; Universidade.

\section{ABSTRACT}

Introduction: According to the WHO (World Health Organization) Quality of Life (QoL) can be defined as 'the perception of the individual about his position in life, in the context of the culture and value system in which he lives and in relation to his/her goals, expectations, standards and concerns". Objective: To investigate the QoL of university professors from a university in Southern Tocantins. Methodology: This was a crosssectional quantitative research conducted with 85 professors from a University of southern Tocantins, Brazil. The data were analyzed through descriptive statistics and according to the WHOQOL-bref correction manual, with data presented in graphs and tables. Results: About their QoL, 75\% declared as good; 14\% neither bad nor good; 9\% rated it as bad nor $2 \%$ pointed out as very good. Final considerations: In summary, the majority did not declare dissatisfaction with their QoL. Except for the Environment score, which was pointed out as low, it is believed that the others are indicators that reflect the good relationship with students, other colleagues and other characteristic aspects of the work process itself.

Keywords: Quality of Life; Professors; University. 


\section{INTRODUÇÃOO}

O termo qualidade de vida (QV) surgiu na literatura a partir de 1975, e apesar de ser um termo corriqueiro no nosso dia a dia ainda é uma expressão sem uma definição clara e objetiva, porém pode-se inferir que abrange "atividades físicas, atividade social e lazer, trabalho, sintomas, renda, cognição, adaptação emocional, autoestima, ansiedade, estresse, atividade sexual, relacionamento interpessoal, impotência, incontinência e satisfação geral com a vida. ${ }^{1}$

De acordo com a Organização Mundial da Saúde (OMS) a QV pode ser definida como "a percepção do indivíduo de sua posição na vida, no contexto da cultura e sistema de valores nos quais ele vive e em relação aos seus objetivos, expectativas, padrões e preocupações". ${ }^{2}$

A QV é uma preocupação progressiva na sociedade, e isso se deve à sua associação com o estado de saúde, sendo considerados até como termos sinônimos, porém trata-se de um termo mais amplo que envolve não só saúde, mas também incorpora o bem-estar físico, mental e social. ${ }^{3}$

Outra grande preocupação está na associação dessas definições com a observação a vários aspectos da vida, em especial o trabalho. Tal aspecto parece representar um fator fundamental para a compreensão da saúde de pessoas e populações na sociedade contemporânea. Neste contexto, os professores formam uma categoria profissional especialmente exposta a rotina de trabalho de desgaste psicológico devido a fatores como carga horária excessiva, baixos salários, condições inapropriadas de trabalho e entraves da própria organização do sistema de ensino. Além disso, trabalhadores da área de serviços, como os profissionais de educação e saúde, policiais e agentes penitenciários que estão em contato do contato direto e excessivo com outros seres humanos - estão mais suscetíveis ao esgotamento mental e burnout. ${ }^{3}$

Os professores universitários transitam no mercado de trabalho submetidos a constantes exigências de qualificação. Suas capacidades físicas, conceituais e atitudinais são acionadas para atingir os propósitos da produtividade acadêmica, o que pode implicar em excessivo estresse de suas funções mentais e comportamentais. ${ }^{4}$

Pereira $(2006)^{5}$ observou que alguns fatores estressantes/estressores aplicáveis à rotina docente incluem atividades administrativas impróprias, com demasiadas funções burocráticas imputadas ao mesmo, escassez de recursos didáticos, regras e técnicas de 
continuidade durante as aulas, carências nas instalações e, principalmente, baixa remuneração insuficiente. $O$ docente lida com diversidade de pessoas e com situações incomuns, entre elas, o grande revezamento de alunos, pressão em busca de atualidades científicas e a quantidade de trabalho além da sala de aula que, nem sempre, é restrito ao espaço acadêmico, o que pode gerar, na maioria dos professores, problemas de saúde emocional que pode comprometer significativamente sua QV.

Diante este cenário suscitou o seguinte questionamento: como estão os escores de qualidade de vida de docentes de uma Universidade do Sul do Tocantins e como os mesmos avaliam sua própria QV?

Acredita-se que os professores avaliam sua QV de forma negativa, refletindo assim em baixos escores de qualidade, que muitas vezes é afetada por uma causa multifatorial, envolvendo questões socioeconômicas e de situação laboral tais como: relacionamento com alunos, relacionamento com demais colegas e coordenação de curso, carga horária de trabalho e infraestrutura da universidade.

A partir do exposto, este estudo objetivou investigar os escores de qualidade de vida de docentes de uma Universidade do Sul do Tocantins.

\section{MATERIAIS E METODOS}

Tratou-se de um estudo transversal de natureza quantitativa, do tipo descritiva, realizada com docentes de uma Universidade do Sul do Estado do Tocantins, Brasil, entre os períodos de maio a julho do ano de 2020.

Por se tratar de uma população finita, com o objetivo de maximizar a representatividade da amostra, a fórmula proposta por Barbetta ${ }^{6}$ foi utilizada para o cálculo amostral, adotando um nível de confiança de 95\% e margem de erro de até 5\%, assim resultou-se no $N$ de 160 docentes como população. No entanto, pelo fato da pesquisa ter sido realizada via formulário eletrônico enviado via e-mail para os docentes, nem todos puderam acessar ou mesmo manifestaram interesse em participar, sendo o $n$ desse estudo de 85 participantes.

Os critérios de inclusão para a participação do estudo foram: ser docente da Universidade de Gurupi; estar no exercício de suas funções no período da coleta de dados; ter endereço eletrônico(e-mail) válido; aceitar a participar da pesquisa; estar ciente, concordando e assinando digitalmente o Termo de Consentimento enviado por e-mail e; responder o questionário por completo. 
O estudo seguiu o que prevê a resolução 466/2012 do Conselho Nacional de Saúde. Assim foi submetida a um Comitê de Ética em Pesquisa com Seres Humanos e aprovada conforme parecer $n^{\circ}: 3.967 .934$. A coleta dos dados foi realizada através do Google Forms no qual foi devidamente exposto os objetivos da pesquisa e o Termo de Consentimento Livre e Esclarecido por completo.

Após a aceitação da participação da pesquisa, os docentes foram direcionados para o questionário sociodemográfico juntamente com o WHOQOL-bref, que é um formulário com 26 questões. O mesmo contém duas questões de caráter geral sobre a qualidade de vida e as outras 24 que visam ponderá-la em quatro domínios, sendo eles: 1 - físico, 2 psicológico, 3 - relações sociais e 4 - ambiente.

As questões são relacionadas a QV geral (percepção da $Q V$ e satisfação com a saúde) e 24 questões relacionadas aos domínios físico (dor e desconforto; energia e fadiga; sono e repouso; mobilidade; atividades da vida cotidiana; dependência de medicação ou tratamentos; capacidade de trabalho), psicológico (sentimentos positivos; pensar, aprender, memória e concentração; autoestima; imagem corporal e aparência; sentimentos negativos; espiritualidade, religião e crenças pessoais), social (relações pessoais; suporte social; atividade sexual) e meio ambiente (segurança física e proteção; ambiente no lar; recursos financeiros; cuidados com a saúde e sociais; oportunidades de adquirir novas informações e habilidades; recreação e lazer; transporte; ambiente físico). Vale ressaltar que todas as questões foram respondidas em uma escala Likert de cinco pontos. ${ }^{7}$

Os dados foram analisados através do método quantitativo por meio de análise descritiva dos dados (frequência relativa/absoluta e porcentagem) através do software SPSS - Statistical Package for the Social Sciences versão 20.0 para Windows.

\section{RESULTADOS E DISCUSSĀO}

Inicialmente os resultados e discussão são apresentados a partir dos dados sociodemográficos e, na sequência, é apresentada a avaliação dos escores a partir dos domínios identificáveis no próprio formulário WOQOL-bref.

Os dados sociodemográficos foram extraídos e categorizados a partir do questionário aplicado aos 85 docentes entrevistados. Os referidos dados são representados a partir das variáveis obtidas nas na tabela a seguir: 
DOI: 10.18606/2318-1419/amazonia.sci.health.v9n1p14-24 Revista Amazônia Science \& Health 2021, Vol.9, № 1
PINTO, KS. DIAS, AR. SALERA, IB. DA SILVA, JB. MARINHO, VL Qualidade de vida em professores universitários: um estudo em uma Universidade do Sul do Tocantins

Tabela 1. Perfil socioeconômico dos docentes

\begin{tabular}{|c|c|c|}
\hline $\begin{array}{l}\text { Variáveis sociodemográficas } \\
(n=85)\end{array}$ & № de docentes & Percentual \\
\hline \multicolumn{3}{|l|}{ Faixa etária } \\
\hline Entre 25 e 30 anos & 8 & $9 \%$ \\
\hline Entre 31 e 40 anos & 35 & $41 \%$ \\
\hline Entre 41 e 50 anos & 21 & $25 \%$ \\
\hline Acima de 50 anos & 21 & $25 \%$ \\
\hline \multicolumn{3}{|l|}{ Sexo dos docentes entrevistados } \\
\hline Masculino & 63 & $75 \%$ \\
\hline Feminino & 22 & $25 \%$ \\
\hline \multicolumn{3}{|l|}{ Estado civil dos docentes } \\
\hline Casado (a) & 58 & $68 \%$ \\
\hline União estável & 4 & $5 \%$ \\
\hline Solteiro(a) & 11 & $13 \%$ \\
\hline Divorciado(a) & 12 & $14 \%$ \\
\hline Viúvo(a) & 0 & $0 \%$ \\
\hline \multicolumn{3}{|c|}{ Número de docentes que possuem filhos } \\
\hline Não & 56 & $34 \%$ \\
\hline Sim & 29 & $66 \%$ \\
\hline \multicolumn{3}{|c|}{ Docentes que professam alguma religião } \\
\hline Não & 8 & $9 \%$ \\
\hline Sim, católica & 36 & $42 \%$ \\
\hline Sim, evangélica & 21 & $25 \%$ \\
\hline Sim, espírita & 14 & $16 \%$ \\
\hline Sim, Outras & 6 & $7 \%$ \\
\hline \multicolumn{3}{|c|}{ Pessoas com quem os docentes residem } \\
\hline Esposa(o)/Companheira(o) & 61 & $72 \%$ \\
\hline Pais & 9 & $11 \%$ \\
\hline Amigos & 0 & $0 \%$ \\
\hline Sozinho & 9 & $11 \%$ \\
\hline Outros & 6 & $7 \%$ \\
\hline \multicolumn{3}{|c|}{ Meio de locomoção que o docente utilizam para irem ao trabalho } \\
\hline Carro & 82 & $96 \%$ \\
\hline Moto & 3 & $4 \%$ \\
\hline Carona & 0 & $0 \%$ \\
\hline Transporte coletivo & 0 & $0 \%$ \\
\hline Táxi/moto-táxi & 0 & $0 \%$ \\
\hline Bicicleta & 0 & $0 \%$ \\
\hline Caminhando & 0 & $0 \%$ \\
\hline \multicolumn{3}{|c|}{ Renda mensal referente à atividade docente } \\
\hline 1 a 2 salários mínimos & 0 & $0 \%$ \\
\hline 2 a 3 salários mínimos & 10 & $11 \%$ \\
\hline 3 a 4 salários mínimos & 16 & $19 \%$ \\
\hline 4 a 10 salários mínimos & 46 & $54 \%$ \\
\hline$\geq 10$ salários mínimos & 13 & $15 \%$ \\
\hline
\end{tabular}

Fonte: dados da pesquisa.

Dos 85 (oitenta e cinco) docentes que participaram da pesquisa, os dados apontaram que a faixa etária variou entre 25 a 50 anos ou mais; a maioria destes (75\%) são do sexo masculino e a minoria (25\%) feminino; sobre o estado civil, a maioria (68\%) são casados e a minoria deles (5\%) possuem união estável; $66 \%$ declararam que possuem filhos e $34 \%$ 
DOI: 10.18606/2318-1419/amazonia.sci.health.v9n1p14-24 Revista Amazônia Science \& Health
PINTO, KS. DIAS, AR. SALERA, IB. DA SILVA, JB. MARINHO, VL

Qualidade de vida em professores universitários: um estudo em uma Universidade do Sul do Tocantins

afirmaram que não; a maioria (42\%) afirmaram serem católicos e a minoria (7\%) professam outro tipo de religião dentre as que não estão no questionário; sobre com quem residem, a maioria (72\%) declarou residir residem com os companheiros/cônjuges e a minoria (7\%) declaram residir com outras pessoas não especificadas no questionário. Quanto à renda mensal obtida com a atividade docente, a maioria (54\%) declaram que ganham entre $4 \mathrm{e}$ 10 salários mínimos e a minoria (11\%) declararam que ganham entre 2 e 3 salários mínimos.

Quanto ao perfil profissional, os docentes responderam questões inerentes ao tempo de exercício profissional, titulação acadêmica, quantidade de disciplinas ministradas na IES e tipo de vínculo profissional. Os dados são expressos na tabela seguinte:

\section{Tabela 2. Perfil profissional dos docentes}

\begin{tabular}{|c|c|c|}
\hline Perfil Profissional $(n=85)$ & № de docentes & Percentual \\
\hline \multicolumn{3}{|l|}{ Tempo de carreira docente } \\
\hline 1 a 3 anos & 12 & $14 \%$ \\
\hline 3 a 5 anos & 9 & $11 \%$ \\
\hline 5 a 10 anos & 17 & $19 \%$ \\
\hline Mais de 10 anos & 47 & $56 \%$ \\
\hline \multicolumn{3}{|l|}{ Titulação dos docentes } \\
\hline Graduado & 2 & $3 \%$ \\
\hline Especialista & 23 & $27 \%$ \\
\hline Mestre (a) & 49 & $57 \%$ \\
\hline Doutor (a) & 9 & $10 \%$ \\
\hline Pós-doutorado & 2 & $3 \%$ \\
\hline \multicolumn{3}{|c|}{ Quantidade de disciplinas ministradas } \\
\hline Entre 01 e 03 disciplinas & 26 & $31 \%$ \\
\hline Entre 03 e 05 disciplinas & 40 & $47 \%$ \\
\hline Entre 05 e 07 disciplinas & 15 & $18 \%$ \\
\hline Mais que 07 disciplinas & 4 & $6 \%$ \\
\hline \multicolumn{3}{|l|}{ Tipo de vínculo profissional } \\
\hline $\begin{array}{c}\text { Efetivo } 40 \text { horas / DE Efetivo } \\
40 \text { horas }\end{array}$ & 16 & $19 \%$ \\
\hline Efetivo 20 horas & 7 & $8 \%$ \\
\hline Substituto 40 horas & 5 & $6 \%$ \\
\hline Substituto 20 horas & 11 & $13 \%$ \\
\hline Não responderam & 46 & $54 \%$ \\
\hline \multicolumn{3}{|c|}{ Docentes que trabalham em outra IES } \\
\hline Sim & 11 & $13 \%$ \\
\hline Não & 74 & $87 \%$ \\
\hline \multicolumn{3}{|c|}{ Docentes que realizam outra atividade profissional além da docência } \\
\hline Sim & 42 & $49 \%$ \\
\hline Não & 43 & $51 \%$ \\
\hline
\end{tabular}

Fonte: dados da pesquisa. 
A maioria dos docentes (56\%) declararam que estão no exercício da docência há mais de 10 anos, outros $19 \%$ estão no exercício da profissão cerca de entre 5 e 10 anos, $14 \%$ entre 1 e 3 anos e $11 \%$ entre 3 e 5 anos.

Sobre sua titulação acadêmica, esta variou entre graduação e pós-doutorado. A maioria dos docentes (57\%) declararam que possuem titulação de mestre, $27 \%$ especialista, $10 \%$ doutorado, $3 \%$ graduado e $3 \%$ possuem titulação de pós-doutorado; dos entrevistados, $47 \%$ declararam que lecionam entre 3 e 5 disciplinas, $31 \%$ entre 1 e 3, $18 \%$ entre 5 e 7 disciplinas e a minoria (6\%) mais que 7 disciplinas.

Quanto ao tipo de vínculo de trabalho, $54 \%$ does entrevistados optaram por não responder a esse questionamento. Já sobre os que responderam 19\% afirmaram que possuem vínculo de docente Efetivo de 40h ou Dedicação Exclusiva (DE), 13\% substituto $20 \mathrm{~h}, 8 \%$ efetivo $20 \mathrm{~h}$ e $6 \%$ substituto $40 \mathrm{~h}$.

Dos entrevistados, destaca-se que a maioria (87\%) afirmaram que não trabalham em outra IES e $13 \%$ afirmaram que sim, trabalham em outra IES; $51 \%$ declarou exercer outra atividade profissional além da docência e $49 \%$ afirmaram que não exercem nenhuma outra atividade profissional além da docência.

Sabe-se que a gama de variáveis diretas e indiretas envolvidas na profissão de docente, bem como o excesso autocobranças, necessidade de se especializar para fins de progressão na carreira e a constante produção acadêmica pertinente à profissão podem ser considerados fatores de risco ao padecimento da categoria.

Em estudo realizado por Oliveira et al (2010) em que se entrevistou docentes da área da saúde, os resultados apontaram que estes não são poupados das demandas globais a que estão submetidos, como tarefas extraclasse, extensa jornada de trabalho, cumprimento de tarefa com prazo curto de tempo, múltiplos empregos e carga horária de sala de aula. ${ }^{8}$

Os autores do referido estudo inferiram que somatório destas demandas propicia o surgimento de efeitos sobre a saúde mental deste grupo ocupacional, sugerindo a necessidade de elaboração de um projeto de intervenção em saúde do trabalhador, para melhorar a condição de trabalho da docente na área da saúde, diminuindo o sofrimento psíquico a que estão expostas, melhorando o grau de satisfação na sua vida familiar, amorosa, social, ambiental e consequentemente em sua atividade profissional, contribuindo para uma melhor qualidade de vida.

Sobre a percepção da QV dos docentes de uma universidade pública do Sul do Tocantins, o gráfico 1 (a seguir) propõe apresentar os respectivos percentuais. 
Gráfico 1. Percepção da qualidade de vida dos docentes

\section{PERCEPÇÃO DA QUALIDADE DE VIDA}

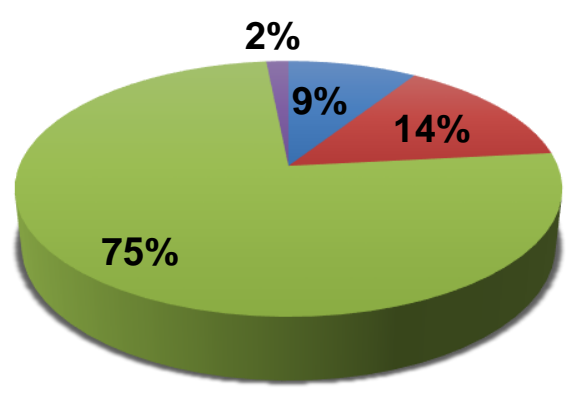

\section{- Ruim $\quad$ Nem ruim e Nem boa $\quad$ Boa $\quad$ Muito boa}

Fonte: dados da pesquisa.

Ao serem questionados sobre como percebem a sua qualidade de vida, a maioria dos docentes (75\%) declararam como boa; $14 \%$ nem ruim e nem boa; $9 \%$ classificaram como ruim e $2 \%$ apontaram como muito boa.

A partir destes dados, infere-se que a maioria dos docentes da universidade pesquisada não declarou insatisfação com sua QV. Este fenômeno refuta a hipótese da pesquisa e os elevados escores podem ser positivos para os gestores da IES, bem como podem servir de instrumentos de gestão de recursos humanos.

Dados semelhantes foram apontados em estudo realizado por Filho, Netto-Oliveira e Oliveira (2012), que analisou a percepção da qualidade de vida geral de docentes e concluiu que a maioria dos entrevistados percebeu sua qualidade de vida como boa ou muito boa, e que se consideraram satisfeitos ou muito satisfeitos com sua saúde. ${ }^{9}$

Tabela 3. Avaliação docentes segundo WHOQOL-bref

\begin{tabular}{ccc}
\hline Domínio & Média & Desvio Padrão \\
\hline Físico & 14,50 & 2,71 \\
Psicológico & 14,63 & 2,28 \\
Relações Sociais & 14,59 & 2,73 \\
Meio Ambiente & 14,26 & 1,75 \\
Auto Avaliação da QV & 14,40 & 3,02 \\
\hline
\end{tabular}


As respostas para todas as questões do WHOQOL-bref são obtidas através de escala tipo Likert de cinco pontos, na qual a pontuação pode variar de 1 a 5 . Tanto os domínios como a qualidade de vida geral são medidos em direção positiva, ou seja, escores mais altos denotam melhor qualidade de vida. Ao somar os escores das 24 facetas e das duas perguntas gerais sobre qualidade de vida, obteve-se um valor médio de 72,38 $\pm 1,93$. Cabe ressaltar que quanto mais alto o escore (máximo de 100), melhor a qualidade de vida.

$\mathrm{Na}$ avaliação da qualidade de vida por meio do questionário WHOQOL-Bref, cada domínio possui pontuação máxima de vinte (20) pontos, onde maiores escores, maior qualidade de vida no domínio. No estudo obteve-se a maior média no domínio Psicológico (14,63\%), seguido do domínio das Relações Sociais (14,59\%), na sequência o domínio Físico (14,50\%) e o domínio Autoavaliação da QV (14,40\%). Ou seja, resultados positivos nestes quatro domínios, exceto o domínio Ambiental (14,26\%).

Em estudo realizado por Klein (2017), que entrevistou docentes de uma IES pública, as conclusões obtidas apontaram que os docentes mostraram-se satisfeitos quanto a Qualidade de Vida no Trabalho (QVT). Para os participantes da pesquisa, a QVT se mostra como sinônimo de um ambiente agradável de trabalho, tanto em relação à estrutura física quanto à harmonia na relação com os colegas, com perspectivas de crescimento profissional e satisfação em relação ao trabalho. ${ }^{10}$

Já o domínio com menor escore foi o ambiental (14,26\%) que diz respeito a segurança física e proteção, ambiente no lar, recursos financeiros, cuidados de saúde e sociais, disponibilidade e qualidade, oportunidades de adquirir novas informações e habilidade, participação em (e oportunidade de) recreação/ lazer, ambiente físico (poluição/ruído/trânsito/clima) e transporte.

Em estudo realizado pelos autores Koetz, Rempel e Périco (2013) numa IES no estado do Rio Grande do Sul, os resultados se assemelharam especificamente a este aspecto, pois a média das respostas aponta para uma boa qualidade de vida em todos os domínios analisados, sendo maior no domínio físico e menor no domínio ambiental. ${ }^{11}$

\section{CONSIDERAÇÕES FINAIS}

O estudo apontou que os docentes da universidade pesquisada avaliam sua QV de forma positiva (boa). Sobre este aspecto, os escores obtidos nos domínios Físico, Psicológico e Relações Sociais podem refletir o bom relacionamento com alunos, demais colegas e outros aspectos característicos do próprio processo de trabalho. 
O domínio Meio Ambiente obteve escores mais baixos, o que pode refletir lacunas pessoais sobre a percepção do docente acerca de sua remuneração, cuidados de saúde, segurança e proteção. Assim, sugere-se que a gestão universitária universidade em questão adote política institucional de QV para realizar acolhimento, proporcione um espaço para constante avaliação e acompanhamento dos seus docentes.

Para que se possa obter maior dimensão acerca da QV das demais universidades do Estado do Tocantins, propõe-se que o estudo seja reproduzido em âmbito territorial para que se proponha políticas de QV para outras IES públicas e contribua para o desenvolvimento das Universidades da região Amazônica.

\section{REFERÉNCIAS}

1. Borine RCC, Wanderley KS, Bassitt DP. Relação entre a qualidade de vida e o estresse em acadêmicos da área da saúde. Est Inter Psicol [Internet]. 2015 [citado 2016 dez. 16];6(1):100-18.

2. Porto LA, Carvalho FM, Oliveira NF, Silvany AM, Araújo TM, Reis EJF, et al. Associação entre distúrbios psíquicos e aspectos psicossociais do trabalho de professores. Rev Saúde Pública 2006; 40:818-26.

3. Davoglio TR, Lettnin CC, Baldissera CG. Avaliação da qualidade de vida em docentes brasileiros: uma revisão sistemática. Pro-Posições. 2015;26(3):145-66.

4. Oliveira, R.R., Silva, I.B., Castro, D.S.P., \& Limongi-França, A.C. (2015). Qualidade de Vida no Trabalho (QVT): Um estudo com professores dos institutos federais. HOLOS, 31(6), 432-447.

5. Pereira RJ, Cotta RMM, Franceschini SCC, Ribeiro RCL, Sampaio RF, Priore SE, et al. Contribuição dos domínios físico, social, psicológico e ambiental para a qualidade de vida global de idosos. Rev Psiquiatr. 2006;28(1):27-38.

6 Barbeta, PA. Estatística aplicada às ciências sociais. 4aㅡ ed. Florianópolis: Editora UFSC, 2002.

7. Fleck MP, Louzada S, Xavier M, Cachamovich E, Vieira G, Santos L, et al. Aplicação da versão em português do instrumento abreviado de avaliação da qualidade de vida WHOQOL-bref. Rev Saúde Pública, 2000; 34:350-6.

8. Oliveira, ERA et al. Gênero e qualidade de vida percebida: estudo com professores da área de saúde. Rev Ciência \& Saúde Coletiva, 2012; 17:741-47.

9. Filho, AO; Netto Oliveira, ER; Oliveira, AAB. Qualidade de vida e fatores de risco de professores universitários. Rev da Educação Física/UEM, 2012; 23:57-67. 
DOI: 10.18606/2318-1419/amazonia.sci.health.v9n1p14-24

Revista Amazônia Science \& Health 2021, Vol.9, № 1
PINTO, KS. DIAS, AR. SALERA, IB. DA SILVA, JB. MARINHO, VL

Qualidade de vida em professores universitários: um estudo em uma Universidade do Sul do Tocantins

10. Klein, LL et al. Qualidade de vida no serviço público: uma avaliação em uma instituição de ensino superior. REAd. Revista Eletrônica de Administração/POA. 2017; 23:317-44.

11. Koetz, L; Rempel, C; Périco, E. Qualidade de vida de professores de Instituições de Ensino Superior Comunitárias do Rio Grande do Sul. Rev Ciência \& Saúde Coletiva, 2013; 18:1019-28. 